6

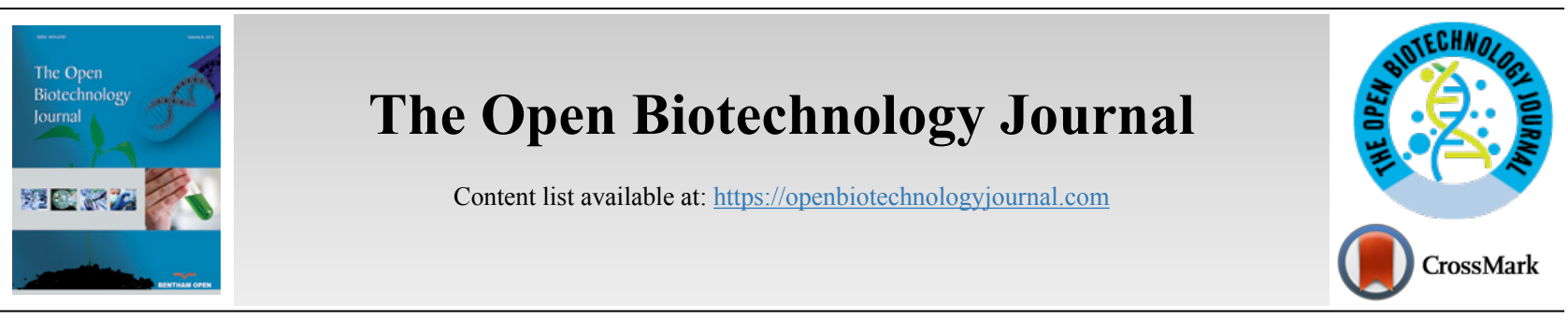

RESEARCH ARTICLE

\title{
De Novo Assembly and Transcriptome Profiling of Ethiopian Lowland Bamboo Oxytenanthera Abyssinica (A.Rich) Munro Under Drought and Salt Stresses
}

\author{
Muhamed Adem ${ }^{1,2,}$, Dereje Beyene ${ }^{2}$, Tileye Feyissa ${ }^{2,3}$, Kai Zhao $^{4}$ and Tingbo Jiang ${ }^{4}$ \\ ${ }^{\prime}$ Department of Forestry, School of Agriculture and Natural Resources, Madawalabu University, P.O. Box 247 Bale Robe, Oromia, Ethiopia \\ ${ }^{2}$ Department of Microbial, Cellular and Molecular Biology, College of Natural and Computational Sciences, Addis Ababa University, P.O. Box 1176 \\ Addis Ababa, Ethiopia \\ ${ }^{3}$ Institute of Biotechnology, College of Natural and Computational Sciences, Addis Ababa University, P.O. Box 1176 Addis Ababa, Ethiopia \\ ${ }^{4}$ State Key Laboratory of Tree Genetics and Breeding, Northeast Forestry University, Harbin, China
}

\begin{abstract}
:
Background:

Bamboos are perennial grasses classified under family Poaceae and subfamily Bambusoideae and are among the fastest growing plants on earth. Despite ecological and economic significances, Ethiopian lowland bamboo (O. abyssinica) lacks global gene expression under abiotic stress.

Methods:

Plastic pot germinated seedlings of $O$. abyssinica were subjected to $200 \mathrm{mM} \mathrm{NaCl}$ and $25 \%$ PEG-6000 (Polyethylene glycol) to induce salt and drought stress, respectively. Using the Illumina sequencing platform, fifteen cDNA libraries were constructed and sequenced to generate the first drought and salt stress transcriptome profiling of the species so as to elucidate genome-wide transcriptome changes in response to such stresses.

Results:

Following quality control, 754,444,646 clean paired-ends reads were generated, and then de novo assembled into 406,181 unigenes. Functional annotation against the public databases presented annotation of 217,067 (53.4\%) unigenes, where NCBI-Nr 203,777, Swissport 115,741, COG 81,632 and KEGG 80,587. Prediction of Transcripts Factors (TFs) have generated 4,332 TFs organized into 64 TF families. Analysis of Differentially Expressed Genes (DEGs) provided 65,471 genes where 569 genes belong to all stresses. Protein families with a higher number of differentially expressed genes include bZIP (49), WRKY (43), MYB (38), AP2/ERF (30), HD-ZIP (25) and MYB related (21).

Conclusion:

In addition to revealing the genome-wide level appraisal of transcriptome resources of the species, this study also uncovered the comprehensive understanding of key stress responsive protein-coding genes, protein families and pathways which could be used as the basis for further studies.
\end{abstract}

Keywords: RNA-Seq, Transcriptome profiling, Differential expressed genes, Drought and salt stress, Transcription factors, O. abyssinica.

\begin{tabular}{l|l|l|l} 
Article History & Received: December 14, 2018 & Revised: February 08, 2019 & Accepted: February 22, 2019
\end{tabular}

\section{INTRODUCTION}

Bambusoideaes are composed of 75 genera and about 1 , 250 species of bamboos distributed in a range of environments from tropical and warm ecosystems to cold regions [1]. On the basis of morphological habit, Bambusoideaes are classified as woody and herbaceous bamboos [2]. Woody bamboos are known by their lignified culms, specialized culm leaves, bisexual flowers, outer ligules on the foliage leaves, complex

* Address correspondence to this author at the Department of Forestry, School of Agriculture and Natural Resources, Madawalabu University, P.O. Box 247 Bale Robe, Oromia, Ethiopia; E-mail: muhamed.adem@aau.edu.et vegetative branching and gregarious monocarpy [3]. Herbaceous bamboos are known by short and more frailly lignified shoots, smaller branches, unisexual flowers, cyclic flowering pattern [3]. Only two species of Bamboos, namely Arundinaria Alpina (K.schum) and Oxytenanthera abyssinica (A.rich) are found in Ethiopia. It is believed that $60 \%$ of African Bamboo resources are found in Ethiopia [4].

O. Abyssinica grows at an altitude range of $700-1,800 \mathrm{~m}$ above sea level in the western part of Ethiopia adjacent to Sudanese Savannah Woodlands. According to FAO and INBAR [5], the major portion of Ethiopia's bamboo (85\%) is 
the O. abyssinica. One of the important features is, it's a hardy species on poor soils in dry vegetation formation. It is most resistant to drought as it tolerates rainfall down to $700 \mathrm{~mm}$ and a high temperature of up to $43^{\circ} \mathrm{C}$ [6]. O.abyssinica has wider applications, including but not limited to housing, fodder, beehives, pulping and paper, flooring and furniture, charcoal, fiber and textile, plywood for truck carriage and molding board for concrete $[7,8]$.

O. abyssinica is clump-forming sympodial bamboo with a strong rhizome up to $10 \mathrm{~cm}$ in diameter grows in pure stands. The culms/stems are grouped into large dense clumps erect and leaning with a length up to $10 \mathrm{~m} \mathrm{[6]}$. The base diameter varies from 3-5 cm. During shooting, culms are solid and later on develop into a small central cavity with a thick culm wall. Due to this, $O$. abysinica is not easier to split for weaving as A. alpina. The stripped leaves are about $20 \mathrm{~cm}$ long and during unfavorable environmental conditions, they have deciduous foliage. There is a morphological difference as some clumps have exceptionally thinner culms and others with much taller and thicker culms among the normal sized ones. Such differences could result from cross-fertilization when flowering. It leads to a variety of seedlings with different growth characteristics of the individual clumps, quite contrary to the monopodial A. alpina [8]. Under conducive natural conditions, it grows at a density of 8000 stems/ha. The above-ground biomass is about 20 tonnes/ha [9].

The basic chromosome number of herbaceous bamboos is $11(\mathrm{x}=11)$, while the basic chromosome number of woody bamboos is believed to be $12(\mathrm{x}=12$.) Woody bamboos have different ploidy levels, the hexaploid $(2 n=6, x=72)$ tropical woody bamboos and the tetraploid $(2 \mathrm{n}=4 \mathrm{x}=48)$ temperate woody bamboos [10].

In response to serious environmental stresses, a series of plant genes with diverse functions are either repressed or enhanced [11 - 13]. Proteins coded with stress-induced genes play a significant role during the occurrence of stresses since they are functional and regulatory proteins. Plant Transcription Factors (TFs) are regulatory proteins which control gene expression in reaction to a range of stresses including drought and salinity. Thus, TFs significantly engage in plant growth and development by regulating defense response and gene regulation networks [14]. In plants like rice [15], and Arabidopsis thaliana [16], many genes have been found to be regulated in common or in particular under drought and salt stress. However, abiotic stress-induced transcriptome studies of $O$. abyssinica have not been undertaken. This study was conducted to unveil the most represented genes, transcript factor families and pathways of the species in response to drought and salt stresses.

\section{MATERIALS AND METHODS}

\subsection{Plant Materials and Treatment}

O. abyssinica seeds used in this research were obtained from Ethiopian biodiversity institute collected from Asossa area, West Ethiopia. Seedlings were germinated and grown under plastic pot at $26^{\circ} \mathrm{C} / 22^{\circ} \mathrm{C}$ (day/night) with $75 \%$ relative humidity, $16 \mathrm{hr}$ photoperiods and $175 \mu \mathrm{mol} / \mathrm{(m}^{2}$. s-1) light intensity. One month grown seedlings with a height range of approximately $35 \mathrm{~cm}$ were transferred to water media for one week to create similar condition before treatment. A total of 90 seedlings (30 seedlings per treatment, 10 seedlings per replication) were used for control, salt and drought stress. The seedlings were treated with $200 \mathrm{mM} \mathrm{NaCl}$ and $25 \%$ PEG-6000 (Polyethylene glycol) to induce salt and drought stress, respectively. The three biological replicates for $24 \mathrm{~h}$ treatment were named as W1, W2, W3 for control, N1, N2, N3 for salt and P1, P2, P3 for drought, while the $48 \mathrm{~h}$ treatment were W4, W5, W6 for control and N4, N5, N6 for salt. Seedlings treated with $25 \%$ PEG-6000 wilted and could not be used for RNA extraction at $48 \mathrm{~h}$. After treatment, 15 independent leaves samples (six control, six salt and three droughts) were collected and directly frozen with liquid nitrogen and stored at $-80{ }^{\circ} \mathrm{C}$ until use.

\subsection{RNA Extraction, cDNA Synthesis, and Sequencing}

RNA was extracted using TRIzol Reagent (Invitrogen, Carlsbad, CA, USA). Total RNA of each sample was qualified and quantified using NanodropLite spectrophotometer (Thermo Scientific Wilmington, Delaware, USA) and Agilent 2100 Bioanalyzer (Agilent Technologies, Palo Alto, CA, USA) with $1 \%$ agarose gel. For library construction, one $\mu \mathrm{g}$ total RNA with RIN value above seven was used. Next-generation sequencing library was constructed according to the manufacturer's protocol (NEBNext Ultra ${ }^{\mathrm{TM}}$ RNA Library Prep Kit for Illumina).

NEBNext Poly(A) mRNA Magnetic Isolation Module (NEB) was used to isolate poly(A) mRNA. The mRNA fragmentation was carried out using NEBNext First-Strand Synthesis Reaction Buffer, while priming was conducted using NEBNext Random Primers. First strand cDNA was synthesized using ProtoScript II Reverse Transcriptase and the second-strand cDNA was synthesized using Second Strand Synthesis Enzyme Mix. The purified double-stranded cDNA by AxyPrep Mag PCR Clean-up (Axygen, Union City, USA) was then treated with End Prep Enzyme Mix to repair both ends and add a dA-tailing in one reaction, followed by a T-A ligation to add adaptors to both ends. Size selection of Adaptor-ligated DNA was then performed using AxyPrep Mag PCR Clean-up (Axygen, Union City, USA), and fragments of $\sim 360 \mathrm{bp}$ (with the approximate insert size of $300 \mathrm{bp}$ ) were recovered. All samples were then amplified by PCR for 11 cycles using P5 and P7 primers, with both primers carrying sequences which can anneal with flow cell to execute bridge PCR and P7 primer carrying a six-base index letting for multiplexing. The PCR products were purified using AxyPrep Mag PCR Clean-up (Axygen, Union City, USA), confirmed using an Agilent 2100 Bioanalyzer (Agilent Technologies, Palo Alto, CA, USA), and quantified by Qubit 2.0 Fluorometer (Invitrogen, Carlsbad, CA, USA). Then libraries with different indices were multiplexed and loaded on an Illumina HiSeq instrument according to manufacturer's instructions (Illumina, San Diego, CA, USA). Sequencing was carried out using a 2x150 bp Paired-End (PE) configuration; image analysis and base calling were conducted by the HiSeq Control Software (HCS) + OLB + GAPipeline-1.6 (Illumina) on the HiSeq instrument. 


\subsection{Data Filtering and Assembly}

Quality assessment of the raw paired-end reads was performed using FastQC v0.11.2 [17]. Pre-processing of raw reads was conducted using Cutadapt v.1.9.1 [18] for adaptor trimming and Sickle v1.33 [19] for quality filtering. Reads obtained after filtering were then used for transcriptome assembly using Trinity v2.2.0 [20]. Trinity works by integrating three different software modules: Inchworm, Chrysalis, and Butterfly execute one after the other to process bulky volumes of RNA-seq reads into full-length transcripts. The duplicated contigs were removed using CD-HIT v4.5.4 [21]. The longest transcripts were taken to be unigenes for functional annotation by identifying nucleotide sequences of all transcripts.

The raw sequencing reads are deposited in the NCBI Sequence Read Archive (SRA) under SRP153816 accession number and the assembled transcripts are also deposited in the Transcriptome Shotgun Assembly (TSA) Database, under GGTK00000000 accession number.

\subsection{Functional Annotation and Classification of De Novo Assembled Unigenes}

The de novo assembled $O$. abyssinica unigenes were annotated through homology searches against public protein databases with an (E-value cut-off of $10^{-5}$ ). The unigene sequences were annotated by using Blast2go [22]. The databases used for annotation include NCBI-Nr, COG, Swissprot, KEGG and GO. GO-Term Finder was used for identifying Gene Ontology (GO) terms that annotate a list of enriched genes with a significant $P$-value $<0.05$. The GO and COG functional classification analyses of all unigenes provide valuable information in predicting possible gene functions and in determining the gene function distribution of $O$. abyssinica unigenes. The KEGG database which deals with genomes, biological pathways, and chemical substances was used to investigate the gene product during plant metabolism and associated gene functions in cellular processes [23].

\subsection{Differentially Expressed Genes (DEGs) Analysis}

For expression analysis, the unigene sequence file as a reference gene file, RSEM v1.2.6 [24] was employed to guesstimate gene and isoform expression levels from the pairend clean data.

FPKM (Fragment per Kilobases per Million reads) was calculated and employed to quantify the expression abundance of transcripts in each sample. For differential expression analysis, the DESeq2 v1.6.3 [25] R program, a model based on the negative binomial distribution was used for determining differential expression from digital gene expression data. A DESeq2 analysis was performed using three combinations: (i) control $v s$. drought, (ii) control $v s$. salt and (iii) drought $v s$. salt. To control the false discovery rate, $P$-value was adjusted by Benjamini and Yekutieli's approach [26]. Genes with $\mid \log _{2}$ Fold change $\mid>1$ and adjusted $P$-value $<0.05$ were treated as differentially expressed.

\subsection{Validation of RNA-Seq Data by RT-qPCR}

To confirm RNA-Seq data by RT-qPCR 40 candidate, unigenes (20 up-regulated and 20 down-regulated) were randomly selected. After removing conserved regions using Expasy (https://www.expasy.org) from the protein sequences, 48 qPCR primers were designed from the full-length cDNA sequences of each unigene using Primer quest tool (supplementary file 1). Total RNA was extracted using TaKaRa MiniVEST Plant RNA Extraction Kit (Takara, Dalian, China). The quality and quantity of total RNA were checked by $1 \%$ agarose gel electrophoresis and NanoDrop 2000c Spectrophotometer (NanoDrop Technologies, Wilmington, DE, USA). The RT-qPCR was performed on an ABI 7500 real-time PCR system (Applied Biosystems, Foster City, CA, USA) using the SYBR Premix Ex Taq ${ }^{\mathrm{TM}}$ kit (Takara, Dalian, China), according to the manufacturer's instructions. The amplification protocol comprised a 5 -minute incubation at $94{ }^{\circ} \mathrm{C}$ then a cycle of $94{ }^{\circ} \mathrm{C}$ for $30 \mathrm{~s}, 60{ }^{\circ} \mathrm{C}$ for the $30 \mathrm{~s}, 72{ }^{\circ} \mathrm{C}$ for $1 \mathrm{~min}$, repeat 35 times, $72{ }^{\circ} \mathrm{C}$ for $10 \mathrm{~min}$, and a final $4{ }^{\circ} \mathrm{C}$ hold. Relative expression of RT-qPCR was calculated 2- $\Delta \Delta$ CT.NTBFTCTTGTTTGA CACCGAAGAGGAG and NTB-R AATAGCTGTCCCT GGAGGAGTTT primers from ERF76 gene that was con-firmed to be used as references for qRTPCR from Moso bamboo [27].

\section{RESULTS}

\subsection{Sequencing, De Novo Assembly and Functional Annotation of $\boldsymbol{O}$. Abyssinica Transcriptome}

The result of Illumina paired-end sequencing and de novo assembly of $O$. abyssinica is presented in Table $\mathbf{1}$ and the length distribution of 406,181 unigenes is illustrated in Fig. (1).

As there was no genomic and transcriptome information published so far on $O$. abyssinica, NCBI-Nr blast search of the unigenes was conducted against other species. Accordingly, Oryza sativa japonica was identified to be the species with the highest homology (41,400 unigenes, 10.2\%), followed by Brachypodium distachyon $(25,886)$ and Setaria italica (21, 881). Other species with considerable matches include Oryza brachyantha $(18,081)$, Zea mays $(15,194)$, Sorghum bicolor (12, 697), Oryza sativa indica (9,504), Aegilops tauschii $(8,162)$, Triticum Urartu $(7,393)$ and Phyllostachys edulis $(6$, $395)$.

From the total 406,181 identified unigenes 217,067 (53.4\%) were successfully annotated using BLASTX searches against the public databases of NCBI-Nr $(203,777,93.8 \%)$, Swissprot $(115,741,53.3 \%)$, COG $(81,632,37.6 \%)$ and KEGG $(80,587,37 \%)$. But the remaining 189,114 (46.6\%) unigenes did not show any significant functional similarity to any of the database explored. The detailed distribution of annotated unigenes to each and multiple databases is shown in Fig. (2).

\subsection{GO and COG Annotation Analysis}

The most over-represented GO terms in response to drought and salt stresses were "binding, 48,000 unigenes", "catalytic activity, 39,000 unigenes" both under molecular function category. "Cell part, 30,000 unigene", "organelle 28,000 unigenes" from Cellular component and "Metabolic 
processes 30,000 unigene", "cellular process 28,000 unigenes" from the biological process was also represented with a significant amount of unigenes (Fig. 3).

Clusters of Orthologous Groups (COG) database annotation searches of 406,181 unigenes have predicted 81,632 unigenes $(20 \%)$ that were assigned into 25 functional categories. The most dominantly represented functional subcategories are "Signal transduction mechanisms" with 12,861 (15.7\%) unigenes followed by "Posttranslational modification, protein turnover, and chaperones" with 10,191 unigenes (12.5\%) which belongs to cellular processing and signaling pathway categories which makes $28 \%$ of the total functional annotations. The third most represented is "General function prediction only" with 9993 unigenes (12.2\%) followed by "Intracellular trafficking, secretion and vesicular transport" with 4968 unigenes (6\%) (Table 2). Among the unigenes categorized into specific COG functional annotations, 4933 unigenes $(6 \%)$ were categorized under "function unknown".

\subsection{Metabolic Pathway Analysis}

To understand the biological functions of the transcriptome genes on the biochemical pathways KEGG functional classification was performed and obtained 80,587 (19.8\%) unigenes involved into 32 KEGG pathway categories. Among

Table 1. Summary of Illumina paired-end sequencing and de novo assembly of $O$. abyssinica.

\begin{tabular}{|c|c|c|}
\hline \multirow[t]{5}{*}{ Sequenced reads } & Number of raw reads & $809,219,680$ \\
\hline & Number of clean reads & $754,444,646$ \\
\hline & Total read length (bp) & $99,601,349,229$ \\
\hline & GC content & 53.72 \\
\hline & Q20 percentage & 97.4 \\
\hline \multirow[t]{5}{*}{ Contigs } & Q30 percentage & 93.09 \\
\hline & Total number & $9,595,574$ \\
\hline & Total length(bp) & $1,101,480,760$ \\
\hline & Mean length(bp) & 374 \\
\hline & Contig N50 (bp) & 566 \\
\hline \multirow[t]{6}{*}{ Unigenes/transcripts } & Total number & 406,181 \\
\hline & Total length (bp) & $240,231,095$ \\
\hline & Mean Length(bp) & 641 \\
\hline & Unigene N50(bp) & 873 \\
\hline & Minimum length(bp) & 201 \\
\hline & Maximum length(bp) & 16651 \\
\hline
\end{tabular}

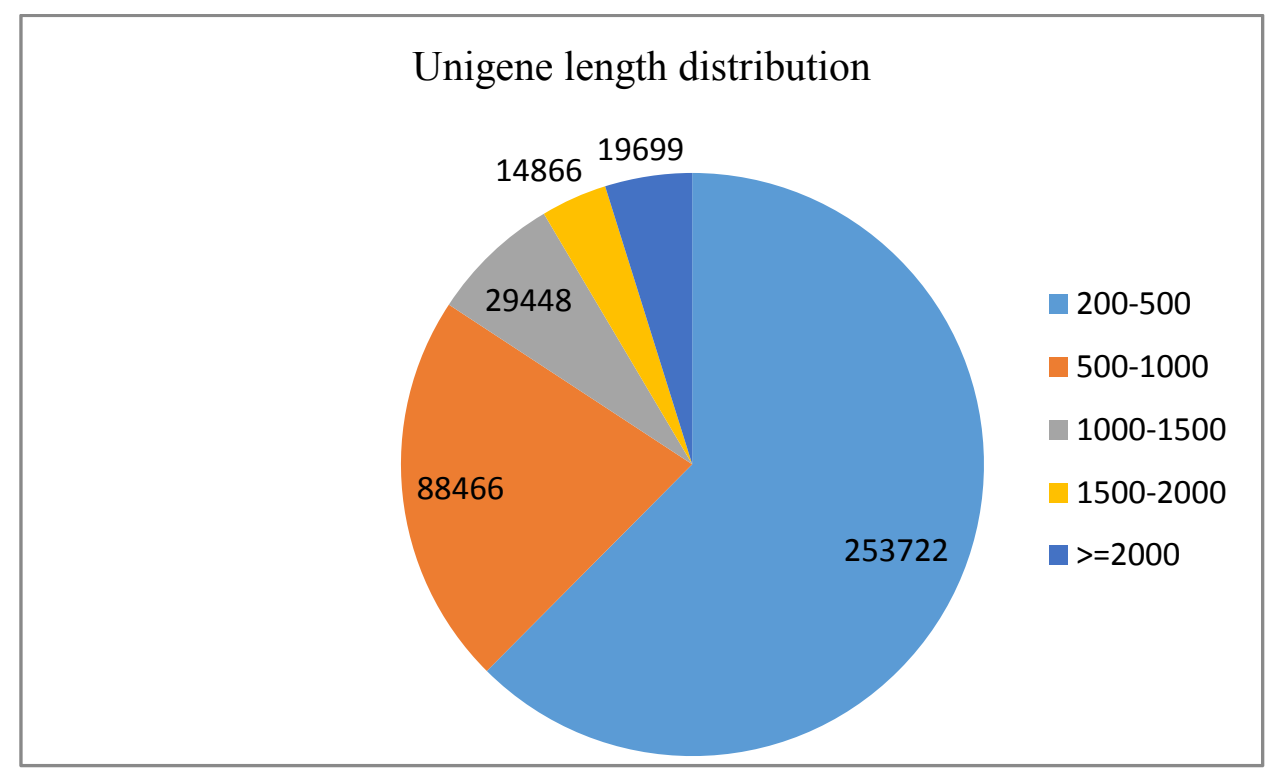

Fig. (1). Unigene length distribution of $O$. abyssinica transcriptome. 


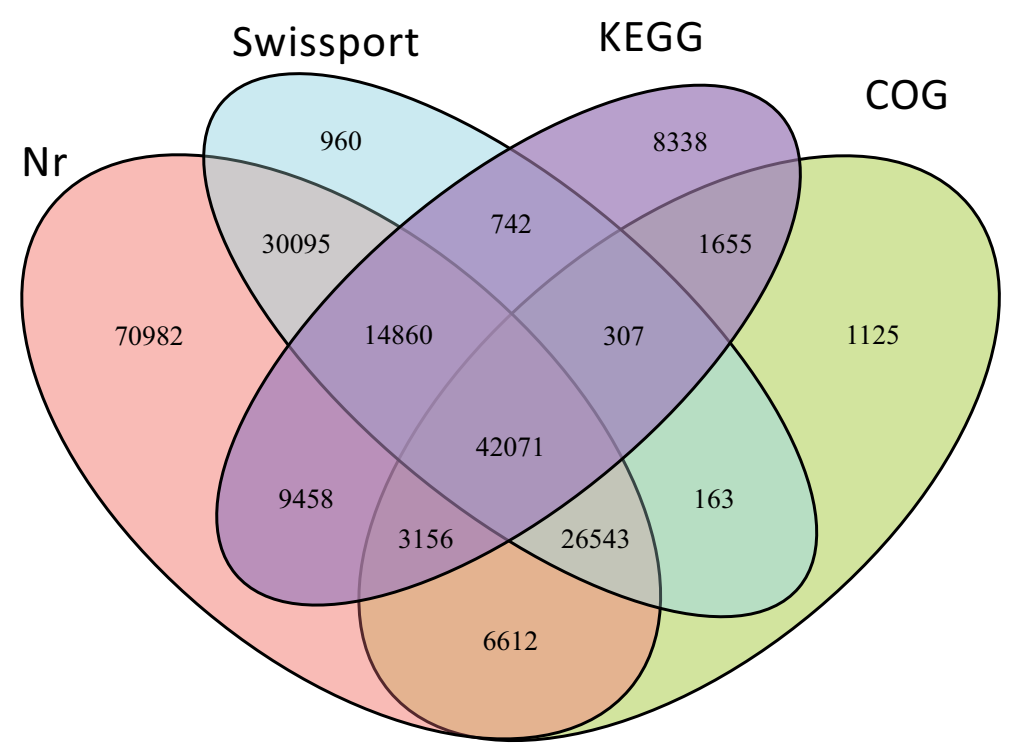

Fig. (2). Venn diagram showing annotation of unigenes.

Table 2. COG functional classifications of Oxytenanthera abyssinica transcripts.

\begin{tabular}{|c|c|c|}
\hline Code & Description & Gene_num \\
\hline & Metabolism & \\
\hline $\mathrm{C}$ & Energy production and conversion & 3803 \\
\hline $\mathrm{Q}$ & Secondary metabolites biosynthesis, transport and catabolism & 2833 \\
\hline $\mathrm{P}$ & Inorganic ion transport and metabolism & 2480 \\
\hline $\mathrm{F}$ & Nucleotide transport and metabolism & 1072 \\
\hline I & Lipid transport and metabolism & 3430 \\
\hline $\mathrm{H}$ & Coenzyme transport and metabolism & 980 \\
\hline G & Carbohydrate transport and metabolism & 4848 \\
\hline \multirow[t]{2}{*}{$\mathrm{E}$} & Amino acid transport and metabolism & 4136 \\
\hline & Information Storage and Processing & \\
\hline $\mathrm{L}$ & Replication, recombination and repair & 2240 \\
\hline $\mathrm{B}$ & Chromatin structure and dynamics & 1179 \\
\hline A & RNA processing and modification & 3702 \\
\hline $\mathrm{K}$ & Transcription & 4928 \\
\hline \multirow[t]{2}{*}{$\mathrm{J}$} & Translation, ribosomal structure and biogenesis & 4987 \\
\hline & Cellular Processing and Signaling & \\
\hline $\mathrm{O}$ & Posttranslational modification, protein turnover, chaperones & 10191 \\
\hline $\mathrm{W}$ & Extracellular structures & 266 \\
\hline $\mathrm{Z}$ & Cytoskeleton & 2343 \\
\hline $\mathrm{M}$ & Cell wall/membrane/envelope biogenesis & 1328 \\
\hline $\mathrm{N}$ & Cell motility & 38 \\
\hline $\mathrm{D}$ & Cell cycle control, cell division, chromosome partitioning & 2066 \\
\hline $\mathrm{T}$ & Signal transduction mechanisms & 12861 \\
\hline $\mathrm{Y}$ & Nuclear structure & 250 \\
\hline $\mathrm{V}$ & Defense mechanisms & 722 \\
\hline \multirow[t]{2}{*}{$\mathrm{U}$} & Intracellular trafficking, secretion, and vesicular transport & 4968 \\
\hline & Poorly characterized & \\
\hline $\mathrm{S}$ & Function unknown & 4933 \\
\hline $\mathrm{R}$ & General function prediction only & 9993 \\
\hline
\end{tabular}




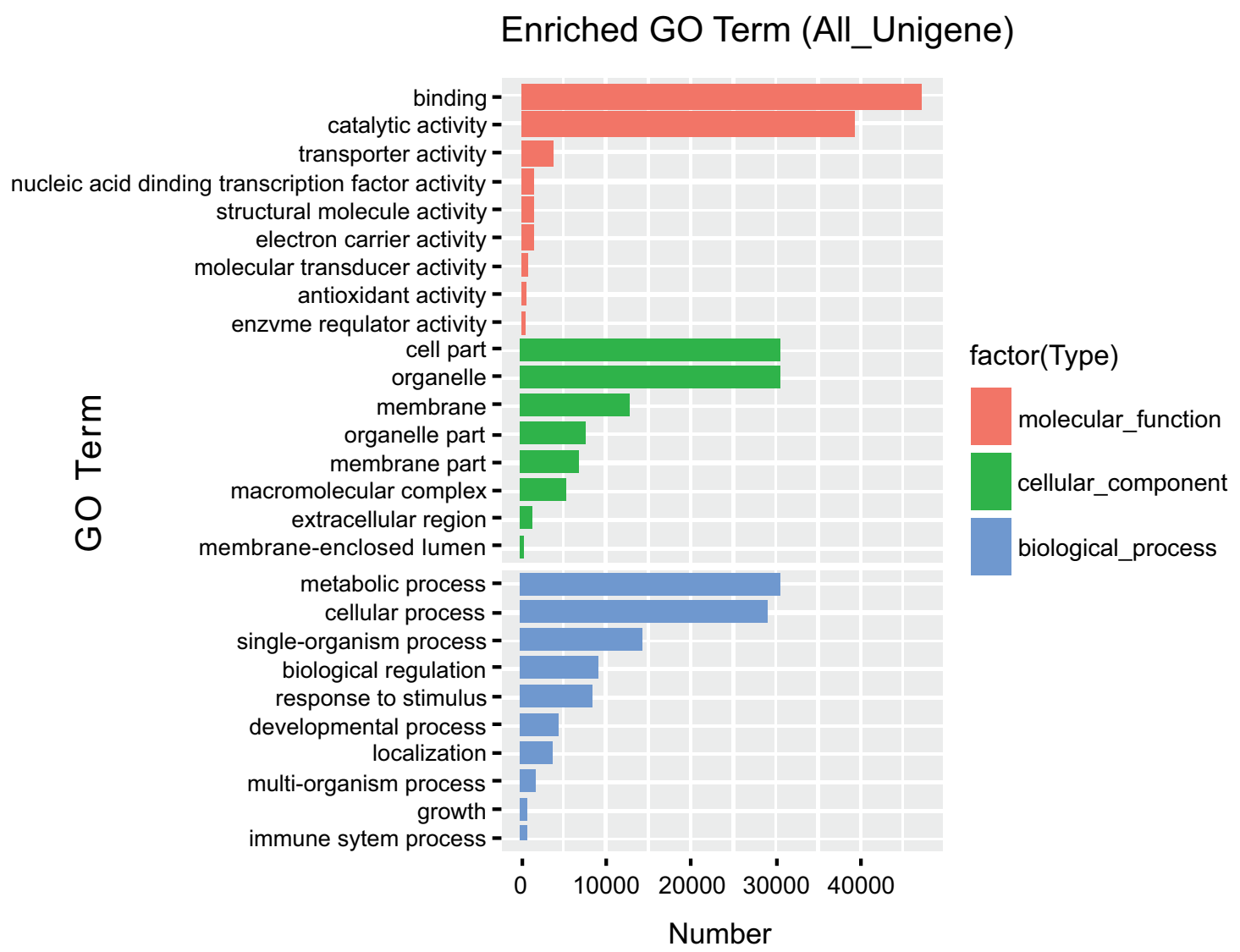

Fig. (3). Gene ontology classification, the vertical axis is the enriched GO terms and the horizontal axis is the number of differential expressed genes in each term. Different colors are used to distinguish between biological processes, cellular components, and molecular functions.

the KEGG pathways categories, signal transduction is the most represented with 25,000 genes $(31 \%)$ followed by carbohydrate metabolism 12,000 genes $(14.9 \%)$. The other pathway categories with a significant number of unigenes include Endocrine system, global and overview maps, translation, folding, sorting, and degradation pathway categories. These pathways have genes ranging from 8,000 to 9,000 genes (Fig. 4).

\subsection{Prediction of Transcription Factors and Protein Family Assignment}

Transcript factors have been exhibited to engage in key tasks in reaction to abiotic stresses by regulating gene expression [28, 29]. For a comprehensive understanding of $O$. abyssinica's gene control and regulation, transcript factors were predicted using family assignment rules established at http://itak.feilab.net/cgi-bin/itak/online_itak.cgi [30]. According to family assignment rules 4,332 Transcript Factors (TFs) were predicted to have active involvement in the regulations of gene expression and these TFs were organized into 64 transcription factors protein families. WD40, NAM/NAC, WRKY, bHLH, AP2/ERF, MYB relate and bZIP, were the most dominant TFs families (Fig. 5). These sequences specific DNA-binding proteins are believed to take part in a decisive role in stress signal transduction pathways. TFs families with proven roles in the regulation of stress response in plants include, NAC, WRKY, AP2/ERF, bZIP, and MYB related [31]. In this study the most represented $\mathrm{TF}$ families with differentially expressed genes (DEGs) includes, bZIP (49),
WRKY (43), MYB (38), AP2/ERF (30), HD-ZIP (25) and MYB related (21), which demonstrates members of these gene families are closely associated with abiotic stress defense [32, 33].

\subsection{Differential Expression Genes (DEGs) Analysis}

The direct expression of a gene's expression level is the abundance of its transcript, the higher the degree of transcript abundance, the higher the gene expression level. DEGs analysis used three combinations: (i) Control vs. drought, (ii) control vs. salt and (iii) drought $v s$. salt. The number of DEGs common and specific between the different stresses were presented in Fig. (6). Clustering analysis was conducted to: (i) calculate the similarity of the data and classify the data according to the similarity so as to cluster together the genes with the same function or close relationship, (ii) to identify the unknown gene function or the unknown function of the known gene and (iii) to infer whether they commonly participate in the same metabolic process or cell pathways.

Differential Expression Genes (DEGs) analysis from this abiotic stress-induced transcriptome study resulted into the expression 65,471 stress-responsive genes, 29,746 up-regulated and 35,725 down-regulated. This indicated that these DEGs are involved in regulating the response to drought and salt stresses. Due to drought stress, a total of 34,719 DEGs were identified, among these 15,681 were up-regulated and 19,038 were down-regulated (Supplementary file 2). Out of 13958 salt 24h stress DEGs, 5994 up-regulated and 7964 down-regulated 


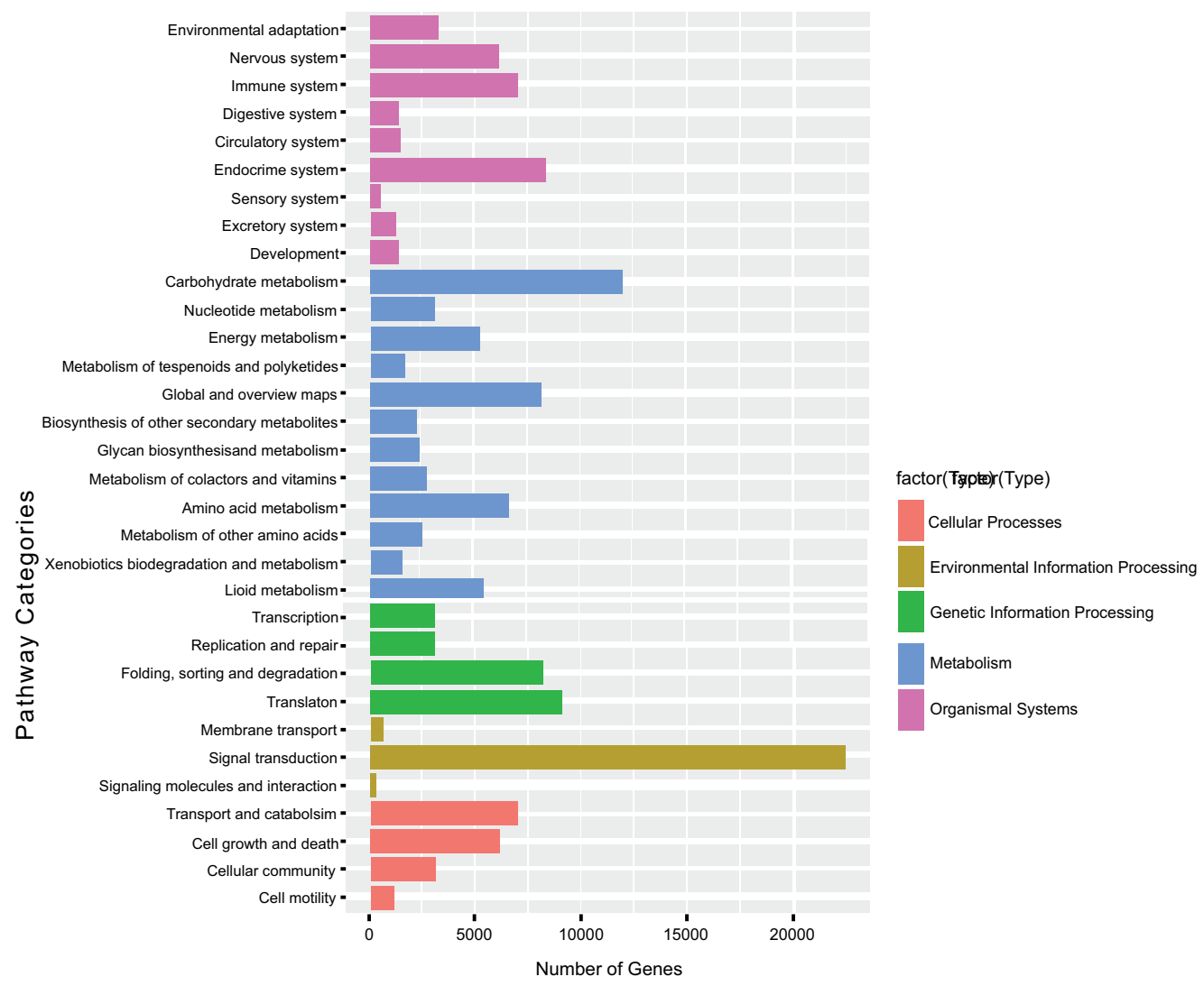

Fig. (4). KEGG classifications, the vertical axis for biological pathways for the six categories; the horizontal line for the number of genes; different colors used to distinguish the biological pathway of a classification.

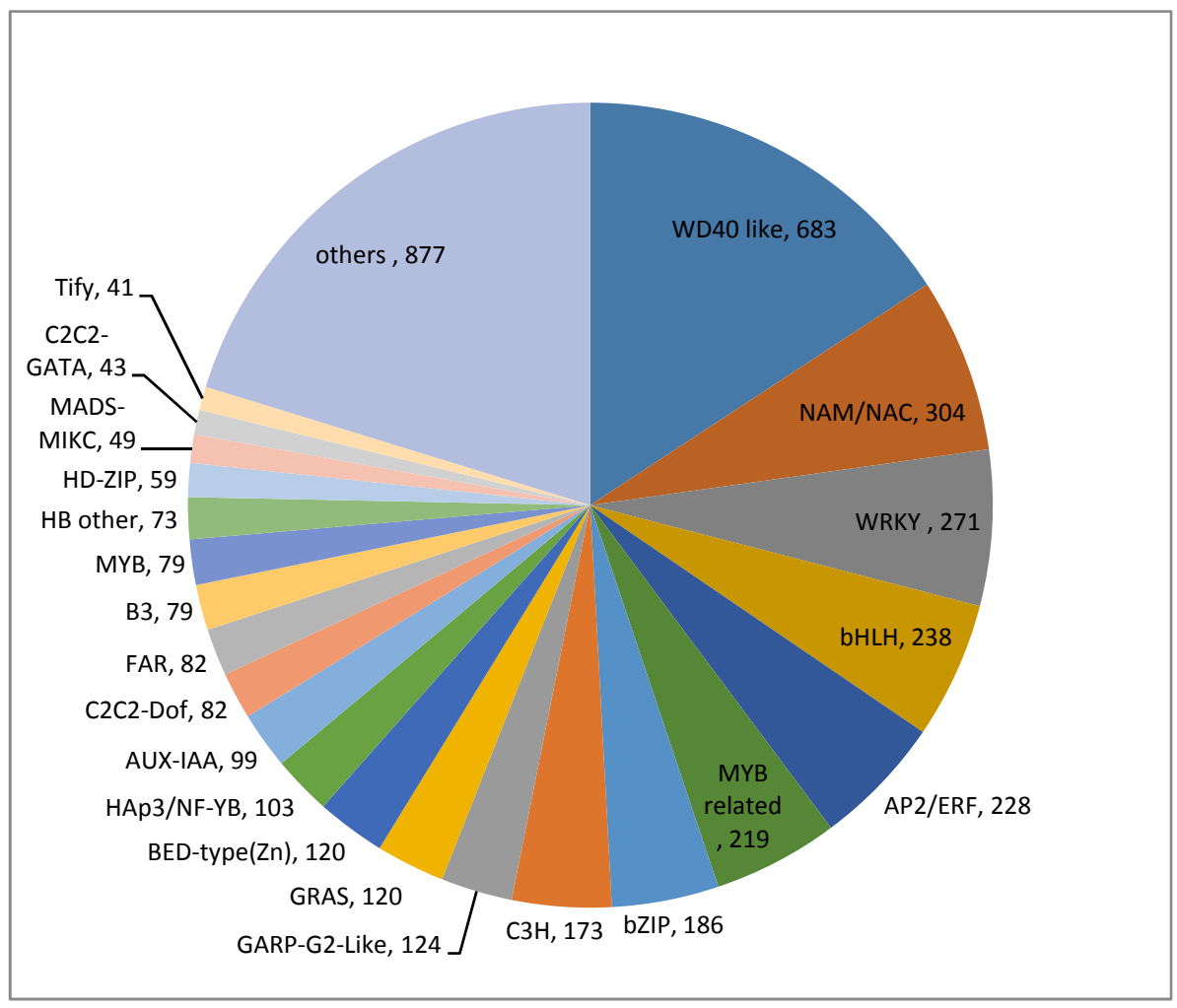

Fig. (5). The most abundant predicted transcription factor families. 


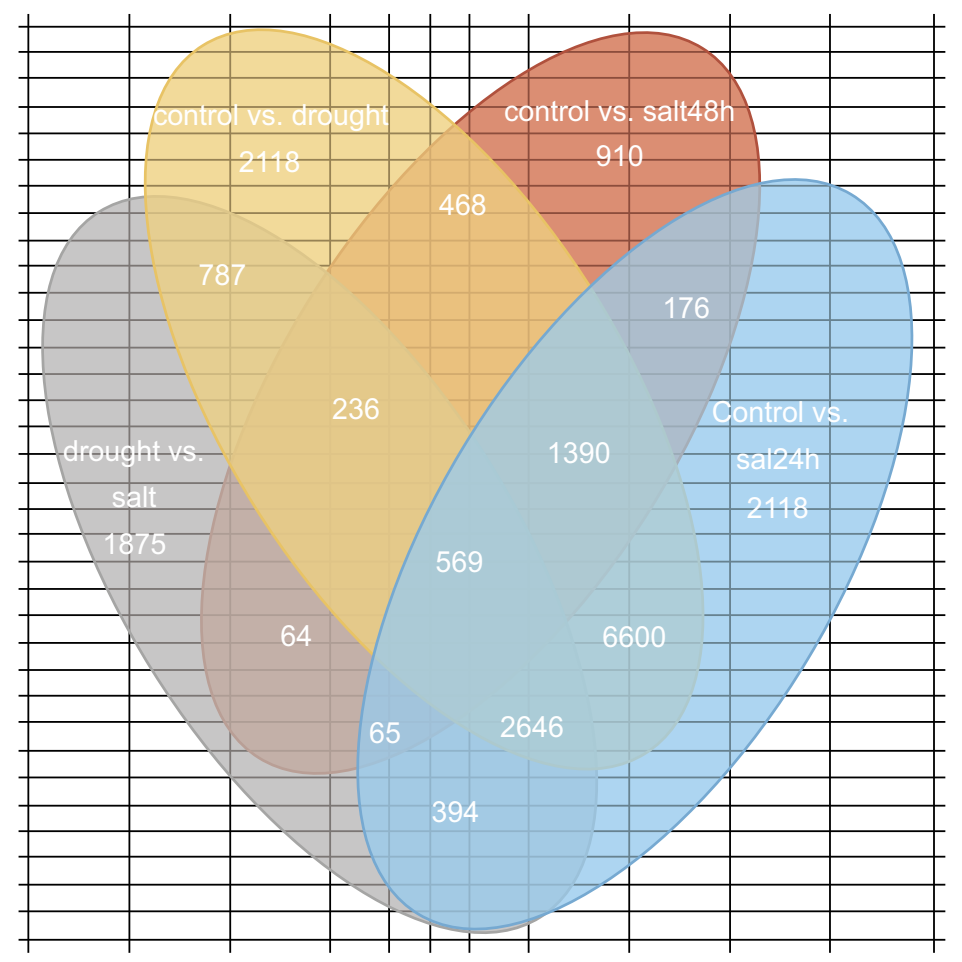

Fig. (6). Venn diagrams showing the number of unique DEGs in each pair of samples, as well as the number of shared DEGs. Treatment groups stand for N1-3 vs. P1-3(drought vs. salt), W1-3 vs. P1-3(control vs. drought), W4-6 vs. N4-6 (control vs. salt 48 h) and W1-3 vs. N1-3(Control vs. salt 24 h).

(Supplementary file 3). Out of 3878 salt 48 h DEGs, 1289 were up-regulated and 2589 were down-regulated (Supplementary file 4). Out of 12,916 droughts vs. salt DEGs, 6,782 were upregulated and 6,134 were down-regulated (Supplementary file 5). Although drought stress has one technical replication the numbers of its DEGs are almost double compared to salt stresses which have two technical replications. This suggests that $O$. abyssinica is more responsive to drought than salt stress. In both drought and salt stresses when compared to control, the numbers of down-regulated DEGs are greater than up-regulated ones. But in the case of drought vs. salt DEGs analysis, up-regulated genes are greater. When we observe DEGs among treatments, genes co-expressed exclusively in each stress includes 15,745 for control $v s$. drought, 2,118 for control vs. salt $24 \mathrm{~h}, 910$ for control $v s$. salt $48 \mathrm{~h}$ and 1,875 for salt vs. drought. But 25,150 DEGs were shared by two or more groups of treatments while 569 were shared by all stress (Fig. 6). DEGs shared by all stresses groups might be blessed in conferring multiple stress tolerance so that those genes might serve as potential candidates for exploring multiple stress tolerance and adaptation.

Out of 65,471 DEGs, $14,258(21.8 \%)$ are functionally annotated. Most importantly $78.2 \%$ of the DEGs were not classified to any functional pathways, which suggests these putative novel genes might be species-specific and these genes might be involved in key pathways regulating in stress adaptation.

Out of 29,746 up-regulated genes, only 7,103 (23.8\%) could be assigned to putative functions. Some genes have a wide range of functions by involving dozens of pathways. For instance, in drought stress, GO: 0000165, GO: 0005524, GO: 0080136, each involved in 97 pathways. In salt $24 \mathrm{~h}$ stress GO: 0080136 (97), GO: 0010099 (37), GO: 2000021 (23). In salt 48 h, GO: 0005739 involved in 18 pathways. In drought $v s$. salt, GO: 0080136 in 97 pathways.

Out of 35,725 down-regulated genes, only 7,155 (20\%) were assigned to functions. The down-regulated genes with the most diverse functions by participating in dozens of pathways includes, in drought stress, GO:0000165, GO:0005634, GO:0042542 and GO:0005524 all under the term mitogenactivated protein kinase involves in 97 pathways, GO:0005952 in 63 pathways. In salt 24, GO: 0005952 (64), GO: 0005509 and GO: 0009536 each in 37 are with the most diverse functions. In salt48h, GO: 0004721 (21) are the most multifunctional genes. In drought vs. salt, GO: 0007264 (41).

\subsection{Validation of RNA-Seq Data by qRT-PCR}

In order to confirm the credibility of the RNA-Seq data, qRT-PCR was conducted. Randomly chosen 20 up-regulated and 20 down-regulated genes were used. To validate the expression levels measured by RNA-Seq, the ratio of expression of selected genes as measured by qRT-PCR was compared to the ratio of expression under drought and salt stress conditions using RNA-Seq. From 40 selected unigenes, only 13 unigenes could be quantified due to specificities of primers. But because of sample replications, these unigenes were quantified from 28 gene samples. The RNA-Seq data have strong positive correlation coefficient value with qRTPCR, since the value of $R^{2}=0.90$. 


\section{DISCUSSION}

The average length of $641 \mathrm{bp}, \mathrm{N} 50$ of $873 \mathrm{bp}$ was more or less comparable with other de novo transcriptome assemblies in other plant species [34 - 38]. The higher ploidy level (hexaploid) nature of $O$. abyssinica might be the reason behind 406,181 unigenes. On the contrary de novo assembled hexaploid hulless Oat has generated 128,414 putative unigenes [39], transcriptome analysis of five hexaploid and allododecaploid Spartina species generated unigenes ranging from 13,054 to 16,002 [40]. In the species similarity search, O. abyssinica showed less similarity with Moso bamboo. This genetic divergence might be due to the existences of more than 1,250 species of bamboos across the globe, differences in speciation time, variation in selection pressure and geographic distances.

When compared to transcriptome analysis of other plants, the 217,067 number of functionally annotated unigenes of $O$. abyssinica were much higher. Although the number is higher, the percentage of annotated unigenes is smaller (53.4\%). For instance, the number of unigenes and annotated percentage of some species includes, 59,814 unigenes (73\%) in drought and cold stresses response of Ammopiptanthus mongolicus, 177, 817 unigenes (94.8\%) in salt response of Spartina alterniflora, 54,125 unigenes $(58.7 \%)$ in desiccation-tolerant moss Syntrichia caninervis, 95,897 unigenes (83.57\%) in Miscanthus sinensis [34, 35, 41, 42].

In $O$. abyssinica, of the total 406,181 unigenes, 217,067 unigenes were functionally annotated. The remaining 189,114 (46.6\%) unigenes did not show any significant functional similarity to any of the database explored. This may be attributed to the lack of references genome of $O$. abyssinica and the highly divergent nature of the unigenes or novel genes that carry out species-specific functions. The GO analysis revealed that binding and catalytic activity from molecular function and metabolic process from the cellular component were represented with a high number of unigenes. More or less similar supporting results were found in transcriptome analysis of Ammopiptanthus mongolicus, Nitraria sibirica and Urochloa decumbens under abiotic stresses [35, 36, 38], this suggests that genes involved in the above GO terms are responsive to abiotic stress.

Only $20 \%$ of unigenes were assigned to COG database and the majority of these unigenes goes to cellular processing and signaling pathway categories. These two dominant subcategories were also with the highest unigenes in a transcriptome analysis of Moss, Tall Fescue and Tobacco [41, 43, 44]. This suggests large numbers of genes in cellular processing and signaling are actively involved in drought and salt stresses response.

Functionally unclassified 4933 unigenes might stand for lineage and/or species-specific genes for adoptive innovation and could be untranslated regions of the transcriptome or novel genes that perform species-specific functions. Signal transduction mechanism at COG and signal transduction at KEGG analysis are represented by the highest numbers of genes, which suggests that both the genes involved and the functional pathways actively respond to stress [35].

The metabolic pathways analysis result suggests that signal transduction and carbohydrate metabolism events are active in $O$. abyssinica response in the face of drought and salt stress. Similar supporting results were obtained from Cotton and Buckwheat transcriptome analysis under abiotic stress [39, 45].

TF families showing up-regulation for both stresses could be targets for studying plant response in the face of unfavorable environmental conditions. Whole genome expression profiling and transcriptome studies in many plants have confirmed the stress responsiveness of various TF families, $\mathrm{C} 2 \mathrm{H} 2$ in Populus trichocarpa [46], NAC in Soybean [47], WD40 in Foxtail Millet [48], WRKY in carrot [44] and Broomcorn millet [49]. In addition to wild plants, many studies have been conducted on the role of $\mathrm{TF}$ families in stress response of transgenic plants, like AP2/ERF conferred stress tolerances in Populus simonii $x$ populous nigra [50], manipulation of specific NAC TFs has conferred stress tolerance in transgenic Rice, Tobacco, Wheat and Arabidopsis [51], AP2/ERF in transgenic Trifolium alexandrinum [50], bHLH in Arabidopsis thailina [52, 53]. The involvements of TF families in diverse abiotic stresses have been reported in different plants [54 - 56].

Analysis of differentially expressed genes has revealed abiotic stress responsiveness of $O$. abyssinica genome. A total of 65,471 DEGs from these 29,746 up-regulated and 35, 725 were down-regulated. DEGs analysis of hexaploid hulless oat has generated 65,000 unigenes [39]. The functionally annotated 14,258 genes proved to be actively involved in diverse pathways activities. As many genes involved in similar functions, the most represented proteins by up-regulated genes include, heat shock $70 \mathrm{kDa}$ protein $1 / 8$, serine/threonine-protein kinase SRK2, protein phosphatase $2 \mathrm{C}$, beta-glucosidase, glutathione S-transferase protein xylosyltransferase, GTPbinding nuclear protein Ran, CTP synthase, adenylate kinase, Delta 1-pyrroline-5-carboxylate Synthetase, glutamine synthetase, Chitinase and glyceraldehyde-3-phosphate dehydrogenase. Majority of the above proteins take part in an important role in the regulation of stress tolerance. Serine-threonine protein kinases are proven to take part in the regulation of signaling cascades and some of these when over-expressed improved stress-tolerance of plants [57]. Glutamine synthetase involves incorporating toxic free ammonium ions into glutamate and glutamine, respectively; thus up-regulation of these genes may be a possible mechanism of stress tolerance [58]. Largely represented up-regulated genes like Delta 1pyrroline-5-carboxylate Synthetase, glyceraldehyde-3-phosphate dehydrogenase and Chitinase play important role in plant defense and enhance resistance and tolerance to drought and salt stresses [35, 59].

The most prevalent proteins encoded from down-regulated DEGs includes, interleukin-1 receptor-associated kinase 4, aldehyde dehydrogenase (NAD+), glutamine synthetase, fructose-bisphosphate aldolase, phosphoenolpyruvate carboxylase, cysteine synthase A, (S)-2-hydroxy-acid oxidase, glutamate synthase (ferredoxin), thioredoxin reductase (NADPH) and light-harvesting complex II chlorophyll $\mathrm{a} / \mathrm{b}$ binding protein.

Among the down-regulated genes, with a significant role in stress tolerances were GABA transporter, arginine, which is one of the precursors of putrescine, glutamate which is the precursor molecule for proline (an osmo-protectant) plays 
important role in plant stress-regulation and stress-tolerance [60]. Phosphatase $2 \mathrm{C}$ family protein also played a significant role in stress tolerance [61]. Receptor protein kinase-like and Glucose-6-phosphate dehydrogenase are also believed to confer stress tolerances in Barley dehydration shock and drought stress [62].

\section{CONCLUSION}

This study has investigated and presented the first comprehensive global transcriptome profiling of $O$. abyssinica under drought and salt stress conditions using the Illumina sequencing platform. The study showed that multiple pathways are involved in drought and salt tolerance. Differentially expressed genes analyses have suggested the stress responsiveness of $O$. abyssinica transcriptome to drought and salt stress since 65,471 genes were differentially expressed. The predicted 4,332 Transcriptions Factors (TFs) organized into 64 TFs families uncovered the most important protein families associated with abiotic stress response. The commonly regulated 569 genes in both abiotic stresses are potential candidates for engineering multiple stress tolerance and adaptation in plants. In general, this transcriptome analysis had identified the key genes, transcription factor families, pathways that can be easily exploited for further transgenesis experiments aiming to investigate genes that are blessed in conferring stress tolerance and adaptation in plants.

\section{ETHICS APPROVAL AND CONSENT TO PARTICI- PATE}

Not applicable.

\section{HUMAN AND ANIMAL RIGHTS}

No animals/humans were used for studies that are the basis of this research.

\section{CONSENT FOR PUBLICATION}

Not applicable.

\section{FUNDING}

We are so much grateful to State Key Laboratory of Tree Genetics and Breeding, Northeast Forestry University, Harbin China, for funding the study.

\section{CONFICT OF INTEREST}

The authors declare no conflict of interest, financial or otherwise.

\section{ACKNOWLEDGEMENTS}

We would like to thank Ethiopian Biodiversity Institute for providing O. abyssinica seeds used for this RNA-Seq study.

\section{SUPPLEMENTARY MATERIAL}

Supplementary material is available on the publishers web site along with the published article.

Supplementary file 1: Primers used for qRT-PCR validation of RNA-Seq data

Supplementary file 2: DEGs analysis for drought stress

Supplementary file 3: DEGs analysis for salt $24 \mathrm{~h}$
Supplementary file 4: DEGs analysis for salt $48 \mathrm{~h}$

Supplementary file 5: DEGs analysis for drought vs. salt

\section{REFERENCES}

[1] Chaomao H, Weiyi L, Xiong Y, Yuming Y. Bamboo for the environment and trade: Environmental Benefits of Bamboo Forests and the Sustainable Development of Bamboo Industry in Western China 2006.

[2] Ramanayake SM, Meemaduma VN, Weerawardene TE. Genetic diversity and relationships between nine species of bamboo in Sri Lanka, using random amplified polymorphic DNA. Plant Syst Evol 2007; 269(1-2): 55-61.

[http://dx.doi.org/10.1007/s00606-007-0587-1]

[3] BPG. Bamboo Phylogeny Group. An updated tribal and sub-tribal classification for the Bambusoideae (Poaceae). Procof the 9th World Bamboo Congress. Gielis J, Potters G, Eds. Antwerp, Belgium. 2012; pp. In: World Bamboo Organization; 3-27.

[4] Yeshambel M, Mengistu U, Getachew A. The role indigenous bamboo species (Yushania alpine and Oxytenanthera abyssinia) as ruminant feed in northwestern Ethiopia. LRRD 2011; 23: 250-8.

[5] (International Network for Bamboo and Rattan) Global Forest Resource assessment Update, Ethiopia Country Report on Bamboo Resources. Addis Ababa, Ethiopia: FAO and INBAR 2005.

[6] Kigomo BN, Kamiri JF. Observations on the growth and yield of Oxythenthera abussinica (A. Rich) munro in plantation. EAfriAgricForJ 1985; 51: 22-9.

[http://dx.doi.org/10.1080/00128325.1985.11663456]

[7] Qisheng Z, Shenxue J, Yongyu T. Industrial Utilization of Bambooo Thechnical Report No 26 International Network for Bamboo and rattan, Beijing, China 2001.

[8] Liese W. Bamboo plantations, The two Bamboos of Ethiopia 2008.

[9] Embaye K. Ecological aspects and resource management of bamboo forests in Ethiopia Doctoral dissertation, ISSN 1401-6230. Swedish University of Agricultural Sciences: Uppsala, Sweden 2003.

[10] Clark LG, Zhang W, Wendel JF. A phylogeny of the grass family (Poaceae) based on ndhF sequence data. Syst Bot 1995; 20(4): 436-60. [http://dx.doi.org/10.2307/2419803]

[11] Lata C, Prasad M. Role of DREBs in regulation of abiotic stress responses in plants. J Exp Bot 2011; 62(14): 4731-48.

[http://dx.doi.org/10.1093/jxb/err210] [PMID: 21737415]

[12] Liu C, Wang B, Li Z, Peng Z, Zhang J. TsNAC1 is a key transcription factor in abiotic stress resistance and growth. Plant Physiol 2018; 176(1): 742-56.

[http://dx.doi.org/10.1104/pp.17.01089] [PMID: 29122985]

[13] De Domenico S, Taurino M, Gallo A, et al. Oxylipin dynamics in Medicago truncatula in response to salt and wounding stresses. Physiol Plant 2019; 165(2): 198-208.

[http://dx.doi.org/10.1111/ppl.12810] [PMID: 30051613]

[14] Pan F, Wang Y, Liu H, et al. Genome-wide identification and expression analysis of SBP-like transcription factor genes in Moso Bamboo (Phyllostachys edulis). BMC Genomics 2017; 18(1): 486. [http://dx.doi.org/10.1186/s12864-017-3882-4] [PMID: 28655295]

[15] Rabbani MA, Maruyama K, Abe H, et al. Monitoring expression profiles of rice genes under cold, drought, and high-salinity stresses and abscisic acid application using cDNA microarray and RNA gelblot analyses. Plant Physiol 2003; 133(4): 1755-67.

[http://dx.doi.org/10.1104/pp.103.025742] [PMID: 14645724]

[16] Seki M, Narusaka M, Ishida J, et al. Monitoring the expression profiles of 7000 Arabidopsis genes under drought, cold and highsalinity stresses using a full-length cDNA microarray. Plant J 2002; 31(3): 279-92.

[http://dx.doi.org/10.1046/j.1365-313X.2002.01359.x] [PMID: 1216 4808]

[17] Andrews S. FastQC: A quality control tool for high throughput sequence data 2010.

[18] Cutadapt removes adapter sequences from high-throughput sequencing reads. EMBnet Journal 2011; 17: 1-10.

[19] Joshi NA, Fass JN. Sickle: A sliding-window, adaptive, quality-based trimming tool for FastQ fles 2011.http://www.citeulike.org /user/mvermaat/article/13260426

[20] Grabherr MG, Haas BJ, Yassour M, et al. Full-length transcriptome assembly from RNA-Seq data without a reference genome. Nat Biotechnol 2011; 29(7): 644-52.

[http://dx.doi.org/10.1038/nbt.1883] [PMID: 21572440] 
[21] Fu L, Niu B, Zhu Z, Wu S, Li W. CD-HIT: Accelerated for clustering the next-generation sequencing data. Bioinformatics 2012; 28(23): $3150-2$.

[http://dx.doi.org/10.1093/bioinformatics/bts565] [PMID: 23060610]

[22] Götz S, García-Gómez JM, Terol J, et al. High-throughput functional annotation and data mining with the Blast2GO suite. Nucleic Acids Res 2008; 36(10): 3420-35.

[http://dx.doi.org/10.1093/nar/gkn176] [PMID: 18445632]

[23] Kanehisa M, Goto S. KEGG: Kyoto encyclopedia of genes and genomes. Nucleic Acids Res 2000; 28(1): 27-30. [http://dx.doi.org/10.1093/nar/28.1.27] [PMID: 10592173]

[24] Li B, Dewey CN. RSEM: Accurate transcript quantification from RNA-Seq data with or without a reference genome. BMC Bioinformatics 2011; 12: 323.

[http://dx.doi.org/10.1186/1471-2105-12-323] [PMID: 21816040]

[25] Anders S, Huber W. Differential expression of RNA-Seq data at the gene level-the DESeq package. Heidelberg, Germany: European Molecular Biology Laboratory (EMBL) 2012.

[26] Benjamini Y, Yekutieli D. The control of the false discovery rate in multiple testing under dependency. Ann Stat 2001; 29: 1165-88.

[27] Fan C, Ma J, Guo Q, Li X, Wang H, Lu M. Selection of reference genes for quantitative real-time PCR in bamboo (Phyllostachys edulis). PLoS One 2013; 8(2): e56573.

[http://dx.doi.org/10.1371/journal.pone.0056573] [PMID: 23437174]

[28] Nuruzzaman M, Sharoni AM, Kikuchi S. Roles of NAC transcription factors in the regulation of biotic and abiotic stress responses in plants. Front Microbiol 2013; 4: 248.

[http://dx.doi.org/10.3389/fmicb.2013.00248] [PMID: 24058359]

[29] Nakashima K, Yamaguchi-Shinozaki K, Shinozaki K. The transcriptional regulatory network in the drought response and its crosstalk in abiotic stress responses including drought, cold, and heat. Front Plant Sci 2014; 5: 170.

[http://dx.doi.org/10.3389/fpls.2014.00170] [PMID: 24904597]

[30] Zheng $\mathrm{Y}$, Jiao C, Sun H, et al. iTAK: A program for genome-wide prediction and classification of plant transcription factors, transcriptional regulators, and protein kinases. Mol Plant 2016; 9(12): 1667-70.

[http://dx.doi.org/10.1016/j.molp.2016.09.014] [PMID: 27717919]

[31] Naika M, Shameer K, Mathew OK, Gowda R, Sowdhamini R. STIFDB2: an updated version of plant stress-responsive transcription factor database with additional stress signals, stress-responsive transcription factor binding sites and stress-responsive genes in Arabidopsis and rice. Plant Cell Physiol 2013; 54(2): e8. [http://dx.doi.org/10.1093/pcp/pcs185] [PMID: 23314754]

[32] Deinlein U, Stephan AB, Horie T, Luo W, Xu G, Schroeder JI. Plant salt-tolerance mechanisms. Trends Plant Sci 2014; 19(6): 371-9. [http://dx.doi.org/10.1016/j.tplants.2014.02.001] [PMID: 24630845]

[33] Zhao H, Lou Y, Sun H, et al. Transcriptome and comparative gene expression analysis of Phyllostachys edulis in response to high light. BMC Plant Biol 2016; 16: 34. [http://dx.doi.org/10.1186/s12870-016-0720-9] [PMID: 26822690]

[34] Liu M, Qiao G, Jiang J, et al. Transcriptome sequencing and de novo analysis for Ma bamboo (Dendrocalamus latiflorus Munro) using the Illumina platform. PLoS One 2012; 7(10): e46766. [http://dx.doi.org/10.1371/journal.pone.0046766] [PMID: 23056442]

[35] Wu Y, Wei W, Pang X, et al. Comparative transcriptome profiling of a desert evergreen shrub, Ammopiptanthus mongolicus, in response to drought and cold stresses. BMC Genomics 2014; 15(1): 671. [http://dx.doi.org/10.1186/1471-2164-15-671] [PMID: 25108399]

[36] Li LQ, Li J, Chen Y, Lu YF, Lu LM. De novo transcriptome analysis of tobacco seedlings and identification of the early response gene network under low-potassium stress. Genet Mol Res 2016; 15(3): 15038599 .

[http://dx.doi.org/10.4238/gmr.15038599] [PMID: 27706558]

[37] Müller M, Seifert S, Lübbe T, Leuschner C, Finkeldey R. De novo transcriptome assembly and analysis of differential gene expression in response to drought in European beech. PLoS One 2017; 12(9): e0184167.

[http://dx.doi.org/10.1371/journal.pone.0184167] [PMID: 28873454]

[38] Salgado LR, Lima R, dos Santos BF, et al. De novo RNA sequencing and analysis of the transcriptome of signalgrass (Urochloa decumbens) roots exposed to aluminum. Plant Growth Regulation 2017; 1; 83(1): 157-70.

[39] Wu Q, Bai X, Zhao W, et al. De novo assembly and analysis of tartary buckwheat (Fagopyrum tataricum Garetn.) transcriptome discloses key regulators involved in salt-stress response. Genes (Basel) 2017; 8(10): 255 [http://dx.doi.org/10.3390/genes8100255] [PMID: 28972562]

[40] Boutte J, Ferreira de Carvalho J, Rousseau-Gueutin M, et al. Reference transcriptomes and detection of duplicated copies in hexaploid and Allododecaploid spartina species. Genome Biol Evol 2016; 8(9): 3030-44.

[http://dx.doi.org/10.1093/gbe/evw209] [PMID: 27614235]

[41] Gao B, Zhang D, Li X, Yang H, Wood AJ. De novo assembly and characterization of the transcriptome in the desiccation-tolerant moss Syntrichia caninervis. BMC Res Notes 2014; 7: 490. [http://dx.doi.org/10.1186/1756-0500-7-490] [PMID: 25086984]

[42] Bedre R, Mangu VR, Srivastava S, Sanchez LE, Baisakh N. Transcriptome analysis of smooth cordgrass (Spartina alterniflora Loisel), a monocot halophyte, reveals candidate genes involved in its adaptation to salinity. BMC Genomics 2016; 17(1): 657.

[http://dx.doi.org/10.1186/s12864-016-3017-3] [PMID: 27542721]

[43] Talukder SK, Azhaguvel P, Mukherjee S, et al. De novo assembly and characterization of tall fescue transcriptome under water stress. Plant Genome 2015.

[http://dx.doi.org/10.3835/plantgenome2014.09.0050]

[44] Li LQ, Li J, Chen Y, Lu YF, Lu LM. De novo transcriptome analysis of tobacco seedlings and identification of the early response gene network under low-potassium stress. Genet Mol Res 2016; 15(3): 15. [http://dx.doi.org/10.4238/gmr.15038599] [PMID: 27706558]

[45] Zhu YN, Shi DQ, Ruan MB, et al. Transcriptome analysis reveals crosstalk of responsive genes to multiple abiotic stresses in cotton (Gossypium hirsutum L.). PLoS One 2013; 8(11): e80218. [http://dx.doi.org/10.1371/journal.pone.0080218] [PMID: 24224045]

[46] Liu Q, Wang Z, Xu X, Zhang H, Li C. Genome-wide analysis of $\mathrm{C}_{2} \mathrm{H}_{2}$ zinc-finger family transcription factors and their responses to abiotic stresses in poplar (Populus trichocarpa). PLoS One 2015; 10(8): e0134753.

[http://dx.doi.org/10.1371/journal.pone.0134753] [PMID: 26237514]

[47] Le DT, Nishiyama R, Watanabe Y, et al. Genome-wide survey and expression analysis of the plant-specific NAC transcription factor family in soybean during development and dehydration stress. DNA Res $2011 ; 18(4): 263-76$

[http://dx.doi.org/10.1093/dnares/dsr015] [PMID: 21685489]

[48] Mishra AK, Muthamilarasan M, Khan Y, Parida SK, Prasad M Genome-wide investigation and expression analyses of WD40 protein family in the model plant foxtail millet (Setaria italica L.). PLoS One 2014; 9(1): e86852.

[http://dx.doi.org/10.1371/journal.pone.0086852] [PMID: 24466268]

[49] Yue H, Wang M, Liu S, Du X, Song W, Nie X. Transcriptome-wide identification and expression profiles of the WRKY transcription factor family in Broomcorn millet (Panicum miliaceum L.). BMC Genomics 2016; 10(17): 343.2016;

[50] Yao W, Zhang X, Zhou B, Zhao K, Li R, Jiang T. Expression pattern of ERF gene family under multiple abiotic stresses in Populus simonii $\times$ P. nigra. Front Plant Sci 2017; 8: 181.

[http://dx.doi.org/10.3389/fpls.2017.00181] [PMID: 28265277]

[51] Shao H, Wang H, Tang X. NAC transcription factors in plant multiple abiotic stress responses: progress and prospects. Front Plant Sci 2015; 6: 902 .

[http://dx.doi.org/10.3389/fpls.2015.00902] [PMID: 26579152]

[52] Abogadallah GM, Nada RM, Malinowski R, Quick P. Overexpression of HARDY, an AP2/ERF gene from Arabidopsis, improves drought and salt tolerance by reducing transpiration and sodium uptake in transgenic Trifolium alexandrinum L. Planta 2011; 233(6): 1265-76. [http://dx.doi.org/10.1007/s00425-011-1382-3] [PMID: 21340699]

[53] Babitha KC, Ramu SV, Pruthvi V, Mahesh P, Nataraja KN, Udayakumar M. Co-expression of AtbHLH17 and AtWRKY28 confers resistance to abiotic stress in Arabidopsis. Transgenic Res 2013; 22(2): 327-41.

[http://dx.doi.org/10.1007/s11248-012-9645-8] [PMID: 22948308]

[54] Wani SH, Singh NB, Devi TR, Haribhushan A, Jeberson SM. Engineering abiotic stress tolerance in plants: Extricating regulatory gene complex. In: Conventional and Non-Conventional Interventions in Crop Improvement. India: CABI New Delhi 2013.

[55] Joshi R, Wani SH, Singh B, et al. Transcription factors and plants response to drought stress: current understanding and future directions. Front Plant Sci 2016; 7: 1029

[http://dx.doi.org/10.3389/fpls.2016.01029] [PMID: 27471513]

[56] Wang H, Wang H, Shao H, Tang X. Recent advances in utilizing transcription factors to improve plant abiotic stress tolerance by transgenic technology. Front Plant Sci 2016; 7: 67.

[PMID: 26904044]

[57] Mao X, Zhang H, Tian S, Chang X, Jing R. TaSnRK2.4, an SNF1-type 
serine/threonine protein kinase of wheat (Triticum aestivum L.), confers enhanced multistress tolerance in Arabidopsis. J Exp Bot 2010; 61(3): 683-96

[http://dx.doi.org/10.1093/jxb/erp331] [PMID: 20022921]

[58] Skopelitis DS, Paranychianakis NV, Paschalidis KA, et al. Abiotic stress generates ROS that signal expression of anionic glutamate dehydrogenases to form glutamate for proline synthesis in tobacco and grapevine. The Plant Cell 2006; 18(10): 2767-81.

[http://dx.doi.org/10.1105/tpc.105.038323]

[59] Magrane M, Consortium U. UniProt Knowledgebase: A hub for protein information. Nucleic Acids Res 2015; 43: 204-12. [doi: 10.1093/nar/gku989].

[http://dx.doi.org/10.1093/nar/gku989]
[60] Shi H, Chan Z. Improvement of plant abiotic stress tolerance through modulation of the polyamine pathway. J Integr Plant Biol 2014; 56(2): 114-21.

[http://dx.doi.org/10.1111/jipb.12128] [PMID: 24401132]

[61] Lee YP, Giorgi FM, Lohse M, et al. Transcriptome sequencing and microarray design for functional genomics in the extremophile Arabidopsis relative Thellungiella salsuginea (Eutrema salsugineum). BMC Genomics 2013; 14: 793.

[http://dx.doi.org/10.1186/1471-2164-14-793] [PMID: 24228715]

[62] Talamè V, Ozturk NZ, Bohnert HJ, Tuberosa R. Barley transcript profiles under dehydration shock and drought stress treatments: A comparative analysis. J Exp Bot 2007; 58(2): 229-40. [http://dx.doi.org/10.1093/jxb/erl163] [PMID: 17110587]

\section{(C) 2019 Adem et al.}

This is an open access article distributed under the terms of the Creative Commons Attribution 4.0 International Public License (CC-BY 4.0), a copy of which is available at: (https://creativecommons.org/licenses/by/4.0/legalcode). This license permits unrestricted use, distribution, and reproduction in any medium, provided the original author and source are credited. 